\title{
SIRT6 keeps pericentromeric transcription in check
}

SIRT6 is a member of the sirtuin family of $\mathrm{NAD}^{+}$-dependent deacetylases and has been demonstrated to recognize acetylated lysines 9 and 56 on histone $\mathrm{H} 3$ (H3K9ac and $\mathrm{H} 3 \mathrm{~K} 56 \mathrm{ac}$, respectively) at telomeres and promoters. SIRT6-mediated deacetylation of these histone residues has been shown to prevent telomeric instability and to silence genes associated with ageing, metabolism and tumour progression. Now Tasselli et al. uncover yet another important role of SIRT6, by showing that its activity inhibits transcription at chromatin regions around centromeres (pericentric regions) and that this transcriptional silencing prevents mitotic errors and cellular senescence.

To uncover new mechanisms through which SIRT6 regulates chromatin states, the authors first investigated its deacetylase activity in vitro and identified $\mathrm{H} 3 \mathrm{~K} 18 \mathrm{ac}$ as a novel substrate. They then verified that SIRT6 activity is indeed necessary for maintaining deacetylation of $\mathrm{H} 3 \mathrm{~K} 18 \mathrm{ac}$ in cells and, interestingly, they observed that deacetylation occurred specifically at pericentric chromatin, but not at other regions.

Typically, pericentric chromatin is silenced, and only very limited transcription takes place in these regions. SIRT6-depleted cells featured markedly increased levels of pericentric transcripts, indicating that SIRT6 functions in pericentric chromatin silencing. Transcriptional repressor KRAB-associated protein 1 (KAP1; also known as TRIM28) was then identified as an effector of this silencing: KAP1 was shown to bind to chromatin in an acetylation-sensitive manner, and $\mathrm{H} 3 \mathrm{~K} 18$ hyperacetylation, resulting from SIRT6 depletion, triggered its release and transcriptional derepression. Altogether, these experiments uncovered a novel, SIRT6-dependent mechanism of pericentric chromatin silencing.

Next, Tasselli et al. set out to investigate the cellular impact of increased pericentric transcription. They observed that upon SIRT6 depletion, cells featured substantial mitotic defects and large genetic aberrations, including chromosomal instability and aneuploidy.

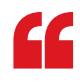

pericentric transcription

... has

negative

effects on

cellular

physiology

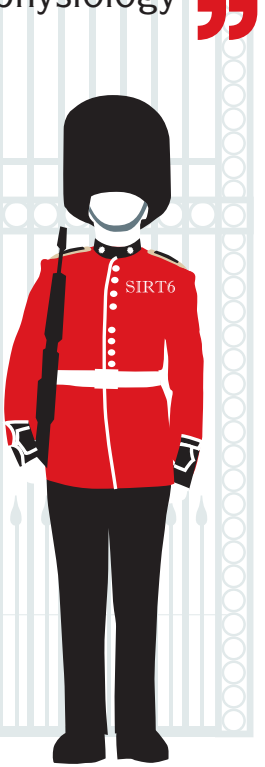

Importantly, these defects could be rescued by short interfering RNA (siRNA)-mediated depletion of pericentric transcripts. Furthermore, SIRT6 depletion or ectopic expression of pericentric transcripts was able to induce cellular senescence.

In sum, this study uncovers an important novel role for SIRT6 in chromatin regulation, whereby SIRT6 limits the expression of pericentric transcripts through specific deacetylation of $\mathrm{H} 3 \mathrm{~K} 18 \mathrm{ac}$ at pericentric chromatin. It also shows that pericentric transcription, when not restrained by SIRT6-mediated deacetylation, has negative effects on cellular physiology, leading to defects typically associated with tumorigenesis and ageing. It would be interesting to investigate how widespread the misregulation of pericentric transcription is in various pathological states in vivo, and to explore whether modulation of SIRT6 expression and/or activity could be used as a potential therapeutic approach for the management of cancer and ageing-associated disorders.

Paulina Strzyz

ORIGINAL ARTICLE Tasselli, L. et al. SIRT6 deacetylates $\mathrm{H} 3 \mathrm{~K} 18 \mathrm{ac}$ at pericentric chromatin to prevent mitotic errors and cellular senescence. Nat. Struct. Mol. Biol. http://dx.doi.org/10.1038/ nsmb.3202 (2016)

FURTHER READING Houtkooper, R. H., Pirinen, E. $\S$ Auwerx, J. Sirtuins as regulators of metabolism and healthspan. Nat. Rev. Mol. Cell Biol. 13 , 225-238 (2012) 